\title{
Port-Hamiltonian Analysis of a Novel Robotic Finger Concept for Minimal Actuation Variable Impedance Grasping
}

\author{
Martin Wassink, Raffaella Carloni and Stefano Stramigioli
}

\begin{abstract}
This paper introduces a novel robotic finger concept for variable impedance grasping in unstructured tasks. A brief literature survey reveals the need for minimal component designs and the benefits of impedance control schemes for interaction tasks such as grasping. The novel robotic finger concept supports these insights by combining three key features: minimal actuation, variable mechanical compliance and full manipulability. This combination of features allows for a minimal component design, while reducing control complexity and still providing required dexterity and grasping capabilities.

The conceptual properties (such as variable compliance) are studied in a port-Hamiltonian framework. The framework proved to be suitable in analyzing and understanding the finger properties, which will be used for future controller design.
\end{abstract}

\section{INTRODUCTION}

Industry shifts towards automated production of customized, small batch and short life-cycle products [1]. Also a new generation of robots is entering the domestic appliances market. In both the household and industrial environment versatile robots are needed to execute a large range of varying tasks in unstructured environments.

Many of these prospected tasks require to interact in mostly human, hence unstructured, environments and to deal with unknown objects. A versatile end-effector, alike the human hand, is one of the critical components for successfully developing this new generation multi-purpose robots. Hence, dexterous robotic hands that have human hand functionality and dimensions are believed to be the required end-effectors. These dexterous robotic hands should be able to grasp (ir)regular objects (pinch and enveloping grasps) and to manipulate objects and fingers (e.g. pre-shaping).

Despite many efforts and breaking research contributions, still current robotic hands present complex designs, housing many (fragile) components, e.g. Salisbury hand [2], Utah/MIT hand [3], Gifu hand [4], UBHand III [5], Karlsruhe hand [6], the DLR hand [7] and the five fingered hand in [8]. They compromise on dimensions, weights, complexity, reliability, functionality and costs. Controlling these devices for stable grasping and manipulation remains a challenge. The number of actuators [1] is a clear source of complexity and weight. Decreasing this number drastically reduces the number of required components, implying

This work has been carried out as part of the FALCON project under the responsibility of the Embedded Systems Institute with Vanderlande Industries as the industrial partner. This project is partially supported by the Netherlands Ministry of Economic Affairs under the Embedded Systems Institute (BSIK03021) program.

\{m.wassink, r.carloni, s.stramigioli $\} @$ utwente.nl, Control Engineering, EE-Math-CS, University of Twente, P.O. Box 217, 7500 AE, Enschede, The Netherlands reduction of weight and energy usage, while robustness improves and cost price benefits as well. For the prospected emerging robotic applications, breakthroughs are needed to get dexterous robotic hands into practice.

The main contribution of this paper is twofold: introducing a novel robotic finger concept for dexterous grasping and presenting a port-Hamiltonian analysis of the concept. Section II gives a brief literature overview. Then, Section III and IV present how lessons learned combine into one robotic concept with three features: minimal actuation, variable mechanical compliance and full manipulability. Section V describes a port-Hamiltonian model, which is used in Section VI for conceptual analysis. This port-Hamiltonian analysis reveals compliance properties and configuration reachability characteristics of the under-actuated finger. Section VII finishes the paper with conclusions and future work.

\section{StATE of THE ART}

This section summarizes parts of current status in grasping research to introduce the novel robotic finger concept in view of these insights. It is not intended to be complete.

\section{A. Dexterity and Grasp Stability}

To achieve dexterity, Salisbury found the minimum number of degrees of freedom (d.o.f.) in a robot hand to be nine [1]. The Salisbury hand and many others have at least three fingers with three d.o.f. each [2] [7] [4] [5] [6] .

Salisbury's force closure rank condition of the grasp matrix categorizes stable and unstable grasps (spatial stability). Montana added a general condition for contact stability [10].

\section{B. Grasp Stiffness and Interaction Control}

Cutkosky and Kao showed the importance of compliance in grasping by computing the overall stiffness matrix (relating external forces to fingertip displacements) of a grasp [11], which gives a direct measure for quasi-static stability. Their analysis showed that unmatched finger stiffness and applied contact forces may destabilize the grip. Interestingly, some stiffness models in robotic grasping appear to effectively model human grasping behavior as well [12].

Besides being useful as grasp stability measure, stiffness analysis is also an interesting topic for interaction control of manipulators. Position and force control strategies tend to destabilize the controller in interaction tasks due to e.g. control loop delays and environment and object uncertainties. In e.g. [13] it was already proposed to intentionally use structural compliance and active stiffness control for grasp 
control. Hogan [14] generalized these approaches and proposed to control the impedance of the robot, i.e. the relation between the interacting forces and velocities [14].

Based on Hogan's ideas, Stramigioli et al. pursued a passive impedance control strategy for stable control of grasping tasks [15]. It was experimentally evaluated in [16].

\section{Programmable Passive Stiffness Components}

Actively controlled impedance can still render unstable behavior due to delay or limited bandwidth, especially when using non-backdriveable mechanics [17]. On the other hand, using direct drive (backdriveable) mechanics reveals other issues such as constant energy consumption for gravity compensation or external overload of the actuator due to impacts. For reasons of safety, passivity and stability, Laurin-Kovitz et al. proposed to control the impedance by incorporating programmable mechanical elements in the robot's drive system [17]. Inspired by human muscles, they developed a device with programmable stiffness and equilibrium position, using antagonistic nonlinear springs. This biomimetic antagonistic principle has been copied many times.

Recently, impedance control with variable stiffness mechanisms was implemented for dexterous grasping [18] [19]. However, the specified desired finger impedance did not include trajectory generation for finger grasp-motion, whereas the impedance control scheme in [15] implements this as part of the desired physical behavior. For safety, miniaturization, stability and energy efficiency, the next DLR hand houses tendon-driven variable stiffness joints by using antagonistic non-linear springs for each joint [20], following [17].

\section{Robot Hand Actuation}

In the 1980's, researchers already predicted actuation challenges in hardware complexity [3]. Recently, Bicchi again emphasized the importance of finding suitable actuation methods and minimizing the number of components [1].

Different actuation methods (e.g. shape memory alloys [21], air muscles [22], fluidic actuators [6]) have been tried of which electromagnetic motors are still the most successful. Due to volume restrictions, most of the grasping devices use remote actuators with tendon transmissions to drive the finger joints from outside the hand without backlash and other gearing difficulties. In [23] another remote actuation approach is shown. The DLR II hand is one of the few exceptions, where direct drive actuators are used in the joints. However, the actuator size is a limiting design factor [20].

Hirose and Umetani introduced an interesting actuation transmission [24] in their 'softgripper'. This under-actuated finger device consists of a pulley routed tendon mechanism. The routing lets the n-d.o.f. finger mechanism naturally conform to any object shape by simply controlling the traction of only one pair of wires per finger. At the cost of dexterity, the number of actuators (and sensors) is reduced significantly, while making it easier to grasp irregular (unknown) objects. Based on these principles, the ARTS Lab produced interesting under-actuated hands, e.g. RTR II [25].

\section{REQUiREMENTS AND DESIGN CONSIDERATIONS}

Dexterous robotic hands should be able to do:

- dexterous finger manipulation for single finger tasks;

- pinch and envelope grasping of (ir)regular objects;

- manipulation of objects.

The main design goals were defined by following the presented observations and considering the prospected applications in unstructured environments:

- low weight;

- energy efficiency;

- robustness (task robustness and reliability);

- human dimensions;

- low cost.

To avoid designs with many actuators, sensors and complex control strategies, the authors propose to focus on designs with a minimal amount of components and variable impedance mechanics that naturally support the hand functions and impedance control strategies. A minimal number of components (especially actuators) allows to optimize dimensions, weight and energy efficiency, while operational robustness and reliability are improved due to the reduction of sensitive components.

Combined control of position and mechanical compliance generally imposes a two d.o.f. control problem per joint. Therefore, a dexterous finger (i.e. three d.o.f.) implies the need for six heavy power actuators per finger, see e.g. [20].

\section{Novel Robotic Finger CONCEPT}

The authors present a novel robotic finger concept that implements the discussed design considerations. The concept, as shown in Figure 1, combines three key features:

1) an antagonistic under-actuated actuation mechanism;

2) series-elastic tendon actuation with non-linear springs;

3) active joint locking mechanisms on the joints.

The concept of minimal components design is implemented by utilizing the under-actuated 'softgripper' design [24], such that to the number of actuators and sensors is reduced. Antagonistic actuation with non-linear springs in the driving tendons gives variable mechanical compliance.

The finger configuration is not fully reachable due to under-actuation. Finger dexterity is restored by locks on each joint: a lock can freeze and release the relative motion of two connected phalanges, without interfering with the free rotating (idle) pulleys on the joints. By means of switching control, the finger configuration space is fully reachable again. Moreover, joint locking does not consume power (theoretically), since motions are constrained to zero velocity. Joint locking is also studied for parallel robot control [26].

\section{Port-Hamiltonian Model}

This section introduces a model of an idealized (rigid bodies, no friction, zero-length springs, perfect locks) realization of the novel concept in a port-Hamiltonian framework and its underlying Dirac structure [9]. The Dirac structure represents the network topology of energy flows in the system. The power ports are interconnected such that power is conserved 


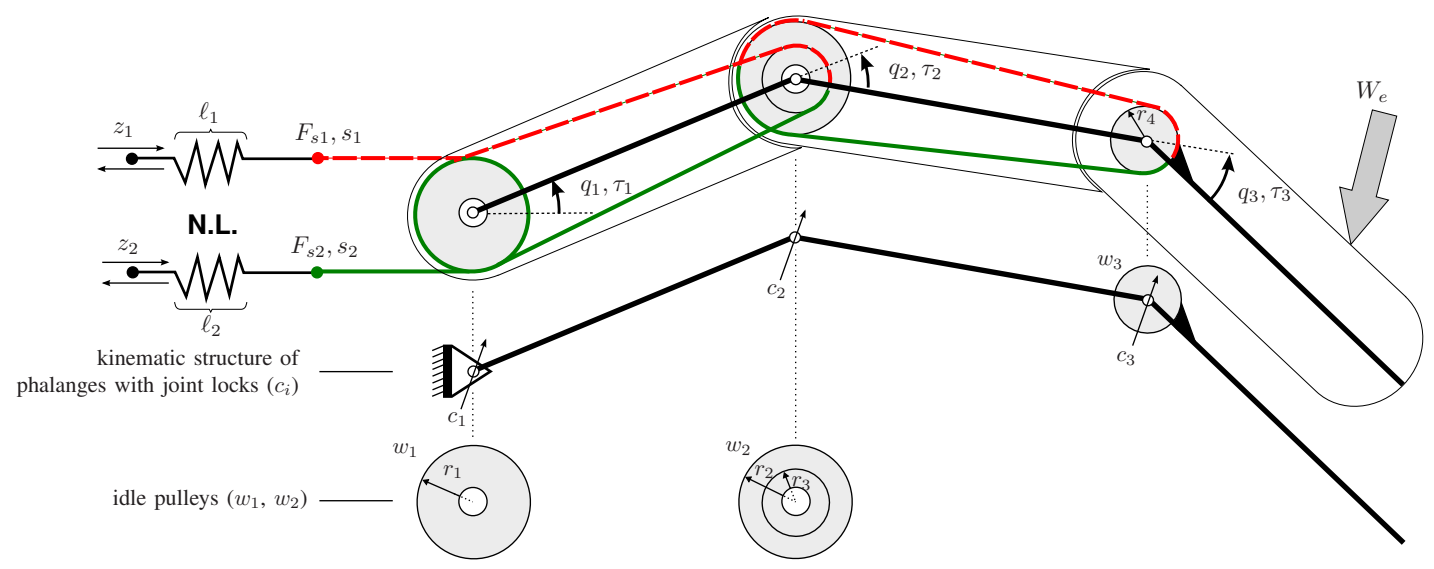

Fig. 1. Conceptual drawing of novel robotic finger: The under-actuated 'softgripper' principle is extended with joint locks and non-linear antagonistic springs (denoted by N.L. with length $\left.\ell_{j}, j \in\{1,2\}\right)$. Pulleys $1\left(w_{1}\right)$ and $2\left(w_{2}\right)$ rotate freely on joint axes. Pulley $3\left(w_{3}\right)$ is fixed to $3^{r d}$ phalanx. Some intuition: The finger is moved by controlling the tendon positions $z=\left(z_{1}, z_{2}\right)^{T}$ in differential mode. Locks $\left(c_{i}\right)$ on each joint can freeze the relative motion of the attached phalanges, i.e. $\dot{q}_{i}=0$, where $q_{i}$ is the relative joint angle of joint $i$ and $\tau_{i}$ the torque on joint $i$. Notice, $w_{1}$ and $w_{2}$ always remain idle. Thus, if a phalanx is constrained by either external interaction or a lock, the other phalanges can still be moved by actuation. In case of object interaction, the phalanges naturally wrap around any shape. The finger configuration is steered by modulating the locks $c_{i}$ and moving $z$. The mechanical compliance of the finger is controlled by driving $z$ in common mode. The externally applied wrench $W_{e}$ represents environment interaction.

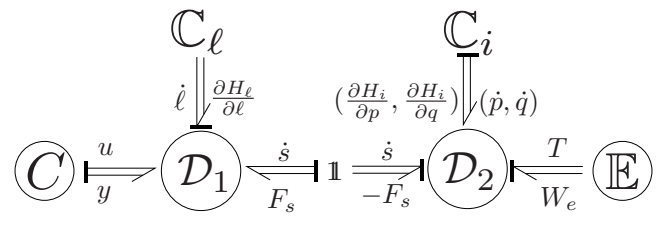

Fig. 2. Generalized bond-graph model of the conceptual finger and its Dirac structures $\mathcal{D}_{1}, \mathcal{D}_{2}$, representing a port-Hamiltonian system with assigned effort causality in the direction of the bar on the bonds. The direction of the bond half-arrow indicates positive power flow.

(as is the case in physical systems) ${ }^{1}$. This framework allows for an energy based, physically consistent, investigation of the conceptual properties for future implementation design and controller synthesis.

\section{A. Port-Hamiltonian Model Without Locks}

The generalized bond-graph model and its Dirac structure of the conceptual finger without locks is depicted in Figure 2. Four different elements are interconnected through their ports on the Dirac structures $\mathcal{D}_{1}, \mathcal{D}_{2}$ :

$\mathbb{C}_{i}: \quad$ Storage of generalized momenta $p=\left(p_{1}, p_{2}, p_{3}\right)^{T}$ of the three phalanges in kinetic energy, given by the Hamiltonian $H_{i}(p, q)=\frac{1}{2} p^{T} M^{-1}(q) p$, where $q=\left(q_{1}, q_{2}, q_{3}\right)^{T}$ is the finger configuration. The port is defined by the dual pair $\left((\dot{p}, \dot{q}),\left(\frac{\partial H_{i}}{\partial p}, \frac{\partial H_{i}}{\partial q}\right)\right)$.

$\mathbb{C}_{\ell}: \quad$ Storage of generalized elongations $\ell=\left(\ell_{1}, \ell_{2}\right)^{T}$ of the two non-linear elastic elements in potential energy, given by some Hamiltonian function $H_{\ell}(\ell)$. The port is defined by the dual pair $\left(\dot{\ell}, \frac{\partial H_{\ell}}{\partial \ell}\right)$.

$\mathbb{E}$ : The environment port is defined by the dual pair $\left(W_{e}, T\right)$. It exerts a wrench ${ }^{2}$ (generalized 6 d.o.f. force) on the finger-tip ( $3^{\text {rd }}$ phalanx) and observes

\footnotetext{
${ }^{1}$ i.e. a generalization of Tellegen's theorem for electrical networks, see also [9] for a formal definition.

${ }^{2}$ See [27] for information on twists and wrenches.
}

the twist (generalized 6 d.o.f. rigid body motions) of the finger-tip.

$C: \quad$ The control port is formed by the input-output dual pair $(u, y)$, where the inputs $u=\left(u_{1}, u_{2}\right)^{T}$ represent the velocities of the tendon actuation positions $z$ (i.e. $u=\dot{z}$ ) and their dual outputs $y=\left(y_{1}, y_{2}\right)^{T}$ are the according tendon forces.

The $\mathbb{1}$-junction interconnects the two Dirac structures through their actuation transmission ports $\left(\dot{s}, F_{s}\right)$, with $\dot{s}$ the time derivatives of the tendon positions $s$ and $F_{s}$ the forces in the tendons.

The Dirac structures $\mathcal{D}_{1}, \mathcal{D}_{2}$ in matrix form are given by:

$$
\begin{aligned}
\mathcal{D}_{1}:\left(\begin{array}{c}
\dot{\ell} \\
F_{s}^{T} \\
y
\end{array}\right)=\left(\begin{array}{ccc}
0 & -\mathbb{I}_{2} & -\mathbb{I}_{2} \\
\mathbb{I}_{2} & 0 & 0 \\
\mathbb{I}_{2} & 0 & 0
\end{array}\right)\left(\begin{array}{c}
\frac{\partial H_{\ell}}{\partial \ell} \\
\dot{s} \\
u
\end{array}\right) \\
\mathcal{D}_{2}:\left(\begin{array}{c}
\dot{p} \\
\dot{q} \\
\dot{s} \\
T
\end{array}\right)=\left(\begin{array}{cccc}
0 & -\mathbb{I}_{3} & -J_{a}^{T} & -J_{q}^{T} \\
\mathbb{I}_{3} & 0 & 0 & 0 \\
J_{a} & 0 & 0 & 0 \\
J_{q} & 0 & 0 & 0
\end{array}\right)\left(\begin{array}{c}
\frac{\partial H_{i}}{\partial p} \\
\frac{\partial H_{i}}{\partial q} \\
-F_{s}^{T} \\
W_{e}^{T}
\end{array}\right)(2)
\end{aligned}
$$

where, due to the $\mathbb{1}$-junction, the sign of $F_{s}^{T}$ is opposite and $\mathbb{I}_{n}$ represents an $n \times n$ identity matrix. Notice that $\dot{p}$ represents the net torques on the joints: $\dot{p}=-\frac{\partial H_{i}}{\partial q}+\tau_{s}-\tau_{e}$.

Furthermore, $J(q)$ (with short notation $J_{q}:=J(q)$ ) defines the tangent map between joint velocities $\dot{q}$ and the twists of the phalanges $T$, called geometric Jacobian [27]:

$$
T=J(q) \cdot \dot{q}
$$

which obviously depends on the finger configuration $q$. The actuation Jacobian $J_{a}$ is the tangent map between the variables $\dot{s}$ and $\dot{q}$ :

$$
\dot{s}=J_{a} \cdot \dot{q}
$$

Notice that $J_{a}$ is constant, hence also the displacements are related equally: $s=J_{a} \cdot q$. Dually, the maps of the dual 


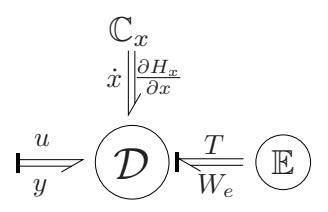

Fig. 3. Reduced and combined total Dirac structure $\mathcal{D}$ with general storage element $\mathbb{C}_{x}$ and state vector $x=\left(p^{T}, q^{T}, \ell^{T}\right)^{T}$.

variables are given by the transpose of the maps in opposite directions, as shown in Eq. 2.

The interconnection of Dirac structures is again a Dirac structure. Therefore, the network representation can be further reduced to one Dirac structure $\mathcal{D}$ (see Figure 3):

$$
\mathcal{D}: \quad\left(\begin{array}{c}
\dot{x} \\
\bar{y}
\end{array}\right)=\left(\begin{array}{cc}
J & g(x) \\
-g^{T}(x) & 0
\end{array}\right) \cdot\left(\begin{array}{c}
\frac{\partial H_{x}}{\partial x} \\
\bar{u}
\end{array}\right)
$$

with skew-symmetric matrix $J$, input-output matrix $g(x)$

$$
J=\left(\begin{array}{ccc}
0 & -\mathbb{I}_{3} & J_{a}^{T} \\
\mathbb{I}_{3} & 0 & 0 \\
-J_{a} & 0 & 0
\end{array}\right), \quad g(x)=\left(\begin{array}{cc}
0 & -J_{q}^{T} \\
0 & 0 \\
-\mathbb{I}_{2} & 0
\end{array}\right)
$$

and the state vector $x=\left(p^{T}, q^{T}, \ell^{T}\right)^{T}$. The total input vector $\bar{u}=\left(u^{T}, W_{e}\right)^{T}$, the total output vector $\bar{y}=\left(y^{T}, T^{T}\right)^{T}$ and the Hamiltonian is given by: $H_{x}(x)=H_{i}(p, q)+H_{\ell}(\ell)$. Hence, the port-Hamiltonian system is represented by:

$$
\begin{cases}\dot{x} & =J \frac{\partial H_{x}}{\partial x}(x)+g(x) \bar{u} \\ -\bar{y} & =g(x)^{T} \frac{\partial H_{x}}{\partial x}(x)\end{cases}
$$

Each bond represents power flow between the ports to which it is connected. Power is defined by the dual product of the power conjugate port variables. Conservation of energy through the external ports $(\bar{u}, \bar{y})$ and the storage port $\left(\dot{x}, \frac{\partial H_{x}}{\partial x}\right)$ is confirmed by recognizing the skew-symmetry of the Dirac structure:

$$
\left(\begin{array}{c}
\dot{x} \\
\bar{y}
\end{array}\right)^{T} \cdot\left(\begin{array}{c}
\frac{\partial H_{x}}{\partial x} \\
\bar{u}
\end{array}\right)=0
$$

Thus, the total rate of energy change in the system is solely determined by the power flows through the external ports.

\section{B. Modeling Locks}

The influence of locks is modeled with constraint equations. The lock inputs are represented by $c=\left(c_{1}, c_{2}, c_{3}\right)^{T}$ with $c_{i} \in\{0,1\}$ where 1 indicates an active lock and 0 an inactive lock. This results in the following port-Hamiltonian model of the finger concept:

$$
\begin{aligned}
& \begin{cases}\dot{x} & =J \frac{\partial H_{x}}{\partial x}(x)+\left(\begin{array}{ll}
g(x) & A(c)
\end{array}\right)\left(\begin{array}{c}
\bar{u} \\
\lambda
\end{array}\right) \\
\left(\begin{array}{c}
-\bar{y} \\
0
\end{array}\right) & =\left(\begin{array}{c}
g(x)^{T} \\
A^{T}(c)
\end{array}\right) \frac{\partial H_{x}}{\partial x}(x)\end{cases} \\
& A^{T}(c)=\left(\begin{array}{cccccccc}
c_{1} & 0 & 0 & 0 & 0 & 0 & 0 & 0 \\
0 & c_{2} & 0 & 0 & 0 & 0 & 0 & 0 \\
0 & 0 & c_{3} & 0 & 0 & 0 & 0 & 0
\end{array}\right)
\end{aligned}
$$

The Lagrangian multipliers $\lambda$ generate the constraint forces $A(c) \lambda$ for the constraints.

\section{PORT-Hamiltonian ANALYsis}

This section analyzes the key-features of the finger concept: controllable compliance and configuration reachability.

\section{A. Conceptual Analysis on Finger-tip Compliance}

The finger compliance $\left(C_{f}\right)$ without actuated locks defines the infinitesimal finger-tip displacement $\delta T$ (i.e. infinitesimal deformation twist) of the finger in response to an externally applied infinitesimal wrench $\delta W_{e}$ around an equilibrium:

$$
\delta T=-C_{f} \cdot \delta W_{e}^{T}
$$

where $\delta T=T \cdot d t=J_{q} \cdot \delta q$ and $\delta q$ is an infinitesimal joint displacement around an equilibrium configuration.

1) External finger-tip compliance condition: Having a finite external finger compliance implies that power can flow from the environment port to the elastic storage $\mathbb{C}_{\ell}$. Hence, a necessary condition for the finger compliance to be finite is the ability to let power flow from the environment to $\mathbb{C}_{\ell}$.

This necessary condition is tested by supplying power $W_{e} T \neq 0$ from the environment with external wrench $W_{e} \neq 0$, while no work is done on the controller input $(u=0)$. In this case, equality must hold for

$$
\frac{d H_{\ell}}{d t}=\left(\frac{\partial H_{\ell}}{\partial \ell}\right)^{T} \dot{\ell}=\alpha \cdot W_{e} T \quad \alpha \in(0,1]
$$

where $\alpha$ indicates that at least a fraction of the total supplied power must flow to the elastic storage element. Naturally, the remaining fraction $(1-\alpha)$ will then flow to $H_{i}$. Applying $u=0$ and substitutions from Eq. 3 give:

$$
-\dot{q}^{T} J_{a}^{T} \frac{\partial H_{\ell}}{\partial \ell}=\alpha \cdot \dot{q}^{T} J_{q}^{T} W_{e}^{T}
$$

Since $\operatorname{Im}\left(J_{a}^{T}\right) \subset \operatorname{Im}\left(J_{q}^{T}\right)$, because of under-actuation, it can be seen that the condition can be satisfied for at least a limited set $W_{s}$, namely:

$$
W_{s}=\left\{W \in s e^{*}(3) \mid J_{q}^{T} W^{T} \in \operatorname{Im}\left(J_{a}^{T}\right)\right\}
$$

Other solutions may exist as well, because of the premultiplication of the row vector $\dot{q}^{T}$. In this case, the necessary condition can be fulfilled and hence finite external finger compliance may exist. This conclusion gives enough ground to continue the compliance analysis.

2) Variable finger-tip compliance: To verify whether the finger compliance can be varied trough the controller port, it is analyzed whether $C_{f}$ can be a function of $u$. To start the analysis, the joint compliance $C_{q}$ is defined by $\frac{\delta q}{\delta \tau}=$ $-C_{q}$, where $\delta \tau$ are the infinitesimal joint torques around some equilibrium. Pre-multiplying with $J_{q}$ and substituting $\delta \tau^{T}=J_{q}^{T} \cdot \delta W^{T}$ (Eq. 3) gives $\delta T=-J_{q} C_{q} J_{q}^{T} \cdot \delta W_{e}^{T}$ and

$$
C_{f}=J_{q} C_{q} J_{q}^{T}
$$

Note that when $J_{q}^{T}$ looses full rank (in a singular configuration), $\delta W_{e} \in \operatorname{ker}\left(J_{q}^{T}\right)$ induces no motion, implying zero compliance (i.e. infinite stiffness). 
The compliance $C_{q}$ is inversely related to the joint stiffness $K_{q}$, defined by $\frac{\delta \tau^{T}}{\delta q}=-K_{q}$. Starting from the linearized force relation of the storage elements

$$
\delta F_{s}^{T}=\frac{\partial^{2} H_{\ell}}{\partial \ell^{2}}(\ell) \cdot \delta \ell
$$

it follows that (using Eq. 3):

$$
\delta \tau^{T}=J_{a}^{T} \cdot \delta F_{s}^{T}=J_{a}^{T} \cdot \frac{\partial^{2} H_{\ell}}{\partial \ell^{2}}(\ell) \cdot\left(-\delta z-J_{a} \delta q\right)
$$

Hence, the joint stiffness $K_{q}$ is found to be:

$$
K_{q}=J_{a}^{T} \cdot \frac{\partial^{2} H_{\ell}}{\partial \ell^{2}}(\ell) \cdot J_{a}
$$

which is the pullback of $\frac{\partial^{2} H_{\ell}}{\partial \ell^{2}}(\ell)$ for the map from $q$ to $s$.

Thus, to be able to vary joint stiffness $K_{q}$ through the controller port, the non-linear springs must be designed such that $\frac{\partial^{2} H_{\ell}}{\partial \ell^{2}}$ is a function of $\ell$, where $\ell$ is known to be a function of the input $u$ (Eq. 3). In that case, naturally, also the joint compliance $C_{q}$ depends on $u$. Therefore it is concluded that the finger-tip compliance $C_{f}$ (Eq. 9) is variable through the controller port if $\frac{\partial^{2} H_{\ell}}{\partial \ell^{2}}$ is a function of $\ell$.

Note that due to the structure of $J_{a}$ due to under-actuation, the matrix $K_{q}$ is not positive definite and has some zero singular values corresponding to some directions of zero stiffness and therefore infinite compliance. By a straight forward singular value decomposition, the directions of infinite compliance can be characterized and correspond to those directions of equal potential of the elastic energy.

\section{B. Conceptual Analysis on Finger Configuration}

This section discusses some configuration reachability properties of the finger concept without actuated locks.

1) Configuration reachability: A necessary condition for the configuration of the finger to be altered by the controller, is the ability to transfer power from the controller port to the inertial storage element such that $\dot{q} \neq 0$. Requiring (partial) power transfer from the controller port to the inertial storage element, with $y^{T} \cdot u \neq 0$, implies requiring:

$$
{\frac{\partial H_{i}}{\partial p}}^{T} \cdot \dot{p}+{\frac{\partial H_{i}}{\partial q}}^{T} \cdot \dot{q}=\beta \cdot y^{T} \cdot u \quad \beta \in(0,1](11)
$$

where $\beta \in(0,1]$ indicates that at least a fraction of the total supplied power must flow to the inertial storage element. After substitution and rewriting, Eq. 11 becomes:

$$
\begin{aligned}
\frac{\partial H_{i}{ }^{T}}{\partial p} \cdot\left(-\frac{\partial H_{i}}{\partial q}+J_{a}^{T} \cdot y\right)+\frac{\partial H_{i}{ }^{T}}{\partial q} \cdot \frac{\partial H_{i}}{\partial p} & =\beta \cdot y^{T} \cdot u \\
y^{T} \cdot J_{a} \cdot \frac{\partial H_{i}}{\partial p} & =\beta \cdot y^{T} \cdot u
\end{aligned}
$$

Thus, to change configuration, $u \in \operatorname{Im}\left(J_{a}\right)$ must be chosen to ensure that the resulting force $y^{T}$ generates equality.
2) Equilibrium configuration: The finger configuration is said to be in static equilibrium if the system has minimal energy w.r.t. the configuration variables. It is found that:

$$
\frac{\partial H_{\ell}}{\partial q}=0 \quad \Rightarrow \quad \frac{\partial H_{\ell}{ }^{T}}{\partial \ell} \frac{\partial \ell}{\partial q}=0 \quad \Leftrightarrow \quad J_{a}^{T} \frac{\partial H_{\ell}}{\partial \ell}(\ell)=0
$$

Hence all spring lengths $\ell$ that render $\frac{\partial H}{\partial \ell}(\ell) \in \operatorname{ker}\left(J_{a}^{T}\right)$ give equilibrium. Furthermore, $\ell=J_{a} q-z$ (see Eq. 3) implies that if $\operatorname{ker}\left(J_{a}\right) \neq \emptyset$ then multiple $q \in \operatorname{ker}\left(J_{a}\right)$ map to the same $\ell$ (for non changing input positions $z$ ) and hence to the same $\frac{\partial H}{\partial \ell}(\ell) \in \operatorname{ker}\left(J_{a}^{T}\right)$.

Thus, if $\operatorname{ker}\left(J_{a}\right) \neq \emptyset$, then the finger equilibrium configuration is not uniquely determined by the states $(\ell)$ of the elastic storage elements. The equilibrium configuration space becomes a higher dimensional space $\left(\operatorname{dim}\left(\operatorname{ker}\left(J_{a}\right)\right)>1\right)$.

\section{Novel Finger Example}

The previous analyses is applied to the proposed conceptual finger. From Figure 1, $J_{a}$ is found to be:

$$
J_{a}=\left(\begin{array}{ccc}
r_{1} & r_{2} & \frac{r_{2} r_{4}}{r_{3}} \\
-r_{1} & -r_{2} & -\frac{r_{2} r_{4}}{r_{3}}
\end{array}\right)
$$

with

$$
\operatorname{ker}\left(J_{a}\right)=\operatorname{span}\left\{\left(\begin{array}{c}
\frac{r_{2}}{r_{1}} \\
-1 \\
0
\end{array}\right),\left(\begin{array}{c}
\frac{r_{2} r_{4}}{r_{1} r_{3}} \\
0 \\
-1
\end{array}\right)\right\}
$$

Hence, the equilibrium configuration space has dimension two and the finger compliance is not finite for all wrenches.

Furthermore,

$$
\operatorname{Im}\left(J_{a}\right)=\operatorname{span}\left\{\left(\begin{array}{c}
1 \\
-1
\end{array}\right)\right\}, \quad \operatorname{ker}\left(J_{a}^{T}\right)=\operatorname{span}\left\{\left(\begin{array}{l}
1 \\
1
\end{array}\right)\right\}
$$

show that inputs $u=\gamma \cdot(1,-1), \quad \gamma \in \mathbb{R}$, i.e. pulling one tendon and releasing the other (differential mode actuation), can change configuration. Whereas, inputs $u=$ $\gamma \cdot(1,1), \quad \gamma \in \mathbb{R}$, i.e. common mode actuation, induce $\frac{\partial H_{\ell}}{\partial \ell}(\ell) \in \operatorname{ker}\left(J_{a}^{T}\right)$, giving no configuration change.

However, common mode actuation does change $\ell$ and hence the finger compliance if the elastic storage functions are designed such that $\frac{\partial^{2} H_{\ell}}{\partial \ell^{2}}$ is a function of $\ell$. Choosing for example a storage function of the form

$$
H_{\ell}(\ell)=\left\{\begin{array}{cc}
\frac{1}{6} k \ell^{3} & \forall \ell \geq 0 \\
0 & \forall \ell<0
\end{array}\right.
$$

gives $\frac{\partial^{2} H_{\ell}}{\partial \ell^{2}}(\ell)=k \ell$, which is clearly a function of $\ell$. Notice, that no energy can be stored in the spring for negative elongations, since the tendons can not push.

\section{Influence of Locks}

Without joint locks, the analysis revealed complications of the finger behavior for $\operatorname{ker}\left(J_{a}\right) \neq \emptyset . A^{T}(c)$ shows that the dynamics of the locked joints reduce to zero. Hence, the dimension of $\operatorname{ker}\left(J_{a}\right)$ is altered, such that by switching $c_{i}$ properly, the whole configuration space of the finger can be reached as desired. Theoretically, locking does not consume power, since the constraints render zero joint velocity. 


\section{E. Grasping and Manipulation Control}

Gesturing, pre-shaping and grasping requires a controller that fully utilizes the mechanical features.

Typical gesturing and pre-shaping control will be done by shaping the compliance equilibrium space of the finger and switching the joint locks such that the finger naturally moves to the desired configuration. For grasping and fixturing objects, an equilibrium configuration space and a desired mechanical compliance have to be set to let the phalanges move to the equilibrium. Instead of reaching this equilibrium, the phalanges naturally adapt their configuration to the object's shape. The contact forces follow from the mechanical compliance and the deviation between the established finger configuration and the equilibrium space.

The equilibrium space and finger compliance are controlled by simple low bandwidth position control for $z$ (see Section VI-C) and joint lock modulation (see Section VID). Only position sensors are needed to measure $q$ and $z$ to derive the tendon stress $F_{s}$ and the joint torques $\tau$. Power can be reduced by using non-backdriveable drives.

\section{CONCLUSIONS AND FUture WORK}

A novel robotic finger concept was introduced for developing multi-fingered dexterous robotic hands with minimal actuation and variable mechanical compliance.

Power flow analysis in a port-Hamiltonian framework supported in verifying the properties of the conceptual finger. It revealed the compliance and reachability properties and showed that the under-actuated driving mechanism endangers these properties. Joint locks were introduced to route power from the controller to the right components to improve reachability.

With this concept, an $n$-fingered hand will have at most $2 n$ large size actuators. Depending on the specific application, the total number of actuators can be reduced by bundling the protagonist and antagonist tendons. Hence, the minimum number of large size actuators for a full dexterous hand would be only two. Future work will study the design of such a hand and the influence of different under-actuated transmission mechanisms and different realizations of locks.

The presented port-Hamiltonian model will be used for controller synthesis. Two controllers are investigated. The impedance based approach of [15] is altered to fully utilize the variable mechanical compliance properties of the finger for grasping. Also a port-based hybrid switching controller is being developed for pre-shaping and gesturing.

\section{REFERENCES}

[1] A. Bicchi, "Hands for dexterous manipulation and robust grasping: a difficult road toward simplicity," IEEE Trans. Robot. Autom., vol. 16, no. 6, pp. 652-662, Dec. 2000 .

[2] J. K. Salisbury and J. J. Craig, "Articulated hands - force control and kinematic issues," Int. J. Robotics Research, vol. 1, no. 1, pp. 4-17, 1982.

[3] S. C. Jacobsen, E. K. Iversen, D. Knutti, R. Johnson, and K. Biggers, "Design of the utah/m.i.t. dextrous hand," in IEEE Int. Conf. Robot. Autom., Apr. 1986.h

[4] T. Mouri, H. Kawasaki, K. Yoshikawa, J. Takai, and S. Ito, "Anthropomorphic robot hand: Gifu hand iii," in Proc. Int. Conf. ICCAS, 2002.
[5] F. Lotti, P. Tiezzi, G. Vassura, L. Biagiotti, C. Melchiorri, and G. Palli, "Ubh 3: A biologically inspired robotic hand," in IEEE Int. Conf. on Intelligent Manipulation and Grasping, Jul. 2004.

[6] A. Kargov, T. Asfour, R. Oberle, H. Klosek, S. Schulz, K. Regenstein G. Bretthauer, and R. Dillmann, "Development of an anthropomorphic hand for a mobile assistive robot," in IEEE 9th Int. Conf. on Rehabilitation Robotics, Jun. 2005.

[7] J. Butterfaß, M. Grebenstein, H. Liu, and G. Hirzinger, "Dlr-hand ii: Next generation of a dextrous robot hand," in IEEE Int. Conf. Robot. Autom., 2001.

[8] I. Yamano and T. Maeno, "Five-fingered robot hand using ultrasonic motors and elastic elements," in Proc. IEEE Int. Conf. Robot. Autom., Apr. 2005.

[9] V. Duindam, A. Macchelli, S. Stramigioli, and H. Bruyninckx, Eds., Modeling and Control of Complex Physical Systems - The PortHamiltonian Approach. Springer, 2009.

[10] D. J. Montana, "The condition for contact grasp stability," in IEEE Int. Conf. Robot. and Autom., Apr. 1991.

[11] M. R. Cutkosky and I. Kao, "Computing and controlling the compliance of a robotic hand," IEEE Trans. Robot. Autom., vol. 5, no. 2, pp. 151-165, Apr. 1989

[12] I. Kao, M. R. Cutkosky, and R. S. Johansson, "Robotic stiffness control and calibration as applied to human grasping tasks," IEEE Trans. Robot. Autom., vol. 13, no. 4, pp. 557-566, Aug. 1997.

[13] J. K. Salisbury, "Active stiffness control of a manipulator in cartesian coordinates," in IEEE Conf. on Decision and Control including the Symposium on Adaptive Processes, vol. 19, Dec. 1980.

[14] N. Hogan, "Impedance control: An approach to manipulation: Part itheory," ASME J. Dyn. Systems, Measurement and Control, vol. 107, pp. 1-7, Mar. 1985.

[15] S. Stramigioli, C. Melchiorri, and S. Andreotti, "A passivity-based control scheme for robotic grasping and manipulation," in Proc. 38th Conf. on Decision \& Control, Dec. 1999.

[16] T. Wimbock, C. Ott, and G. Hirzinger, "Analysis and experimental evaluation of the intrinsically passive controller (ipc) for multifingered hands," in IEEE Int. Conf. Robot. Autom., May 2008.

[17] K. F. Laurin-Kovitz, J. E. Colgate, and S. D. R. Carnes, "Design of components for programmable passive impedance," in IEEE Int. Conf. Robot. Autom., Apr. 1991.

[18] T. Wimbock, C. Ott, A. Albu-Schaeffer, A. Kugi, and G. Hirzinger, "Impedance control for variable stiffness mechanisms with nonlinear joint coupling," in IEEE/RSJ Int. Conf. on Intelligent Robots and Systems. IEEE, Sep. 2008.

[19] S. Wolf and G. Hirzinger, "A new variable stiffness design: Matching requirements of the next robot generation," in IEEE Int. Conf. Robot. Autom., May 2008.

[20] M. Grebenstein and P. van der Smagt, "Antagonism for a highly anthropomorphic hand-arm system," Advanced Robotics, vol. 22, no. 1, pp. 39-55, 2008.

[21] A. D. Price, A. Jnifene, and H. E. Naguib, "Design and control of a shape memory alloy based dexterous robot hand," Smart Materials and Structures, vol. 16, no. 4, pp. 1401-1414, Jul. 2007.

[22] F. Rothling, R. Haschke, J. J. Steil, and H. Ritter, "Platform portable anthropomorphic grasping with the bielefeld 20-dof shadow and 9-dof tum hand," in IEEE/RSJ Int. Conf. on Intelligent Robots and Systems, Nov. 2007.

[23] K. Koganezawa and Y. Ishizuka, "Novel mechanism of artificial finger using double planetary gear system," in IEEE/RSJ Int. Conf. on Intelligent Robots and Systems, Sep. 2008.

[24] S. Hirose and Y. Umetani, "The development of soft gripper for the versatile robot hand," Mechanism and Machine Theory, vol. 13, pp. 351-359, 1978.

[25] B. Massa, S. Roccella, M. C. Carrozza, and P. Dario, "Design and development of an underactuated prosthetic hand," in IEEE Int. Conf. Robot. Autom., May 2002.

[26] N. Pavlovic, R. Keimer, and H.-J. Franke, "Adaptronic revolute joints for parallel robots based on simultaneous quasi-statical axial and radial clearance adjustment," in ASME Int. Design Engineering Technical Conferences \& Computers and Information in Engineering Conference, Aug. 2008.

[27] S. Stramigioli, Modeling and IPC Control of Interactive Mechanical Systems, A Coordinate-Free Approach, 1st ed. Springer-Verlag, 2001. 